\title{
TOWARDS FOCUSED MARKETS OF RESOURCES FOR AGILE VIRTUAL ENTERPRISE INTEGRATION
}

\author{
Maria Manuela Cunha \\ Instituto Politécnico do Cávado e do Ave, Portugal, mcunha@ipca.pt \\ Goran D. Putnik \\ Universidade do Minho, Portugal, putnikgd@eng.uminho.pt \\ Paulo Ávila \\ Instituto Politécnico do Porto, Portugal, pavila@dem.isep.ipp.pt
}

\begin{abstract}
Due to the complexity of the search process, the dynamics inherent to the integration of resources in Agile/Virtual Enterprises $(A / V E)$ is not compatible with the optimal search of resources in the universal or global domain of potential resources, designated as Market of Resources. So, we defend that the future paradigm of $A / V E$ integration will gain efficiency if the search for the optimal takes place in a restricted domain, subset from the universal domain, called Focused Market of Resources. This approach implies two distinct processes: 1) a process of identification and filtering of the focused market, i.e. a focused domain, from the universal, global domain, and 2) the search process for resources over the focused domain. These two processes are separated "in time and space" meaning that the first process is an "off-line" preparatory process, before the demand for resources appears. The identified focused market is then submitted to the second process, an "on-line" effective selection process, at the time the demand for resources appears, based on a search algorithm to find the optimal resources match to integrate the $A / V E$. In this paper we propose the existence of Market of Resources, as an global environment and domain for the $A / V E$ integration, and the Focused Markets of Resources as the privileged environment to the efficient integration of Agile/Virtual Enterprises.
\end{abstract}

\section{INTRODUCTION}

Buying and selling goods and services across national borders is hardly a new phenomenon; international trade has been in existence for ever. In the past few decades however, the volume of international trade has risen dramatically. Increasingly, managers in industries ranging from telecommunications, pharmaceuticals, and apparel to retailing, copiers, and automobiles are looking abroad for resources and sales, even as they daily face overseas business entering their own markets (Prahalad, 1987).

Enterprises are becoming global businesses, organised in networks, integrated in supply chains and integrating different units such as shop-floors, logistic centres, 
warehousing infrastructures, etc. Globalisation is the present paradigm. To the absolute dimension of globalisation corresponds not just the idea of global markets, but the concept of global networks, in a global economy, using the full potential of the information and communication technologies. The advances on information and communication technologies (ICT) allowed the process to be extended through continents, creating markets and systems not only global and distributed, but also virtual.

More than twenty years ago, several authors realised the importance of information and knowledge and the wide range of implications,. Their theories and concepts of a post industrial or information economy are still an important basis for research on the impacts of these developments on the society as a whole. These changes demands re-engineering /adaptation within enterprises, as well as revision of corporate strategies.

The combination of the shorter life span of new products, rapid technological developments as well as frequent changes in demand increases the need for a more efficient production to keep competitiveness. Thus, competitiveness is a main requisite of enterprises, whose satisfaction requires the definition of new organisational concepts either of enterprises, or of production systems, with extremely high performances.

There are several factors determining the performance of the new organisational models. One of the most important factors is the organisation capability of fast adaptability or fast reconfigurability i.e. flexibility. In any case the reconfigurability, as a part of flexibility, implies the new resources search, which we will allocate to the task to be performed, in order to satisfy the new circumstances (the new tasks, optimisation of old tasks, "deadlocks", etc.).

However, the traditional organisational model for the problem of reconfigurability, uses the own resources existing within the organisation ${ }^{2}$, i.e. company. The set of the own resources of the company itself represents the resources selection domain. As the selection domain is of a relatively limited, small size, in general, it cannot provide the desired performances neither for actual products nor for new products.

To solve the problem of the lack of resources that could bring to the company a competitive advantage, the company searches for co-operation with other companies simply buying components, subcontracting other companies or creating strategic or

1 In the concept of Flexible Manufacturing Systems, the flexibility is defined as "a capability of the (manufacturing) system to adapt to the new tasks (i.e. to reconfigure, i.e. to reprogram itself in order to satisfy the demand in an optimal way) without interruption of the production (manufacturing) process". So "adaptability", or "reconfigurability", is necessary but not sufficient conditions for flexibility. Any system is possible to adapt but we seek for adaptation of the system so fast that the production process will be not affected. Based on this premises "fast adaptability" or "fast reconfigurability" are synonyms for "flexibility".

We also state that the "flexibility" is either equal (synonym) to "agility" or is a part of it (flexibility $\supseteq$ agility).

2 In traditional organisation the combined and/or integrated process planning (PP) and production planning and control (PPC) carry on the reconfigurability of the manufacturing system. The operation design, as the PP function, and the manufacturing operations scheduling, as the PPC function, both include a machine tool selection, i.e. resources selection, on the existing workshop as the resource selection domain.

Similar processes are carried on for the business processes where the reconfigurability is termed as a company "re-organisation". 
joint-venture associations. This experience is already known for a long time but in the last years new factors have emerged that brought a different view on the enterprise organisation:

- a requisite for an extremely high dynamics of the company reconfigurability (due to increased competition), i.e. in search for new resources, through companies association creation, cooperation, etc.

- globalization of economy, making possible to consider the resources that exist all over the world.

As a consequence of the above mentioned factors, the actual concept of competitive enterprise implies, among many other characteristics, the ability of flexible access to the optimal resources, as well as the capability to manage all business and manufacturing functions, independently of distance. These are the main ideas under the concepts of Distributed Manufacturing Systems. We define a "distributed manufacturing system or enterprise" as a manufacturing system or enterprise which performances does not depend on the physical distance between the enterprise elements (Putnik G., 1998) ${ }^{3}$. An implementation of the distributed manufacturing system, in terms of its spatially distributed elements, is the wellknown Global Manufacturing Systems concept (Mitsuishi, 1992).

The models satisfying the requisite of fast reconfigurability are the Agile Enterprise and Virtual Enterprise ones ${ }^{4}$ (Agile/Virtual Enterprise - A/V E). and Agile Enterprise/ Manufacturing or Virtual Enterprise/ Factory (Goldman, 1995; NIIIP, 1996; Kim, 1990; Onosata, 1993; Putnik, 1997).

\section{FRAMEWORK FOR A MARKET OF RESOURCES}

Accordingly with several definitions, virtual enterprises are defined as "agile" enterprises, i.e. as enterprises with integration and reconfiguration capability in useful time (in "real time"), integrated from "cells" or independent enterprises, primitive or complex, with the aim of taking profit from a specific market opportunity. After the conclusion of that opportunity, the virtual enterprise dissolves and a new virtual enterprise is integrated, or it reconfigures itself in order to achieve the necessary competitiveness to respond to another market opportunity.

For the independent, primitive or complex enterprises, or companies, candidates to integrate an agile or virtual enterprise, we will use the designation "resource", or "resources", in the sense that, from the point of view of the virtual enterprise, these enterprises represent the candidate "resources" for integration.

In other words, a virtual enterprise is a temporary and dynamic association of independent resources ("cells", enterprises), primitive or complex, that brings to the association, i.e., to the virtual enterprise, its best practices and core competencies to

3 The most referred interpretations of Distributed Manufacturing Systems are related to the distributed control of manufacturing system, based on Multi-Agent System model, and to the spatial distribution of manufacturing system functions - physical components. Our concern in this text is the second interpretation of distributed manufacturing systems, the spatial distribution of manufacturing system components, which does not imply distributed control in terms of Multi-Agent Systems.

4 Although we state that there is a difference between the Agile and Virtual enterprise concepts, in the context of the present work, we will not address it, as our main concern is the flexibility given by the characteristic of fast reconfigurability intrinsic to both models. 
achieve the highest competitiveness. On the other side, to obtain the best experiences and competencies, it is desirable, or it is necessary, that as many as possible primitive or complex resources ("cells", enterprises) concur to the integration in the (virtual) enterprise. The best case occurs if to the integration in the enterprise concur the independent resources ("cells", enterprises) from the universal domain ${ }^{5}$. This requisite implies that the candidate resources to integrate an association, to fulfill a specific market opportunity, i.e., to integrate a virtual enterprise, are globally distributed and inter-connected using ICT, to enable the negotiation capability, (to integrate the association or virtual enterprise) and operation (of the virtual enterprise, as they got into it) in efficient, effective and real time way. The global distribution of candidate resources for integration on virtual enterprises implies the concept of "distributed" enterprises".

Three relevant requisites are identified in relation with the process of $A / V E$ design or integration:

1) access to independent candidate resources to integrate a virtual enterprise, negotiation process between them and its integration;

2) global distribution of the independent candidate resources to integrate a virtual enterprise or of the independent resources participating in a virtual enterprise;

3) minimisation of the integration time.

The first characteristic implies the existence of a market of independent candidate resources for integrating a virtual enterprise. This market role is:

a) to provide the environment and technology and the corresponding procedure protocols, i.e. "the open system architecture" (of the resource market) for the efficient access to resources, efficient negotiation between them and its efficient integration;

b) to provide a domain for selection of participant resources in a virtual enterprise, large enough to assure the best, or near the best options ${ }^{7}$, i.e., to provide the global domain to competitive access to any potential resource.

The second characteristic implies the utilisation of the advanced information and communication technologies to the operation of the independent resources market, i.e., technologies providing technical conditions to efficiently accede to the globally distributed resources, efficient negotiation between them and its efficient integration.

The third characteristic is necessary in order to provide the flexibility as high as possible, i.e. the reconfigurability as fast as possible.

In order to achieve the highest performances (productivity, costs, response time, quality, etc.) of the $\mathrm{A} / \mathrm{V} \mathrm{E}$ design and operation processes, it is of the highest interest to consider their automation, total or partial (Computer Aided). The process

5 From the implementation point of view, the universal domain is the biggest domain that we could refer, or to which we could have access. Due to the present process of economy globalisation, along with the tremendous development of information and communication technologies (ICT) it is obvious that the universal domain today corresponds virtually to the global domain.

6 Distributed enterprises do not imply virtual enterprises. We may say that distributed enterprises are an intermediate step on the development and implementation of the virtual enterprise concept. In the same way, we can imagine several cases where distributed enterprises take advantage when compared with virtual enterprises, i.e., cases where the application of the virtual enterprise concept does not apply. But, from the other side the virtual enterprise will have the biggest advantage if includes the distributed enterprise features.

7 "Near the best" means optimised, not necessarily optimal, implying an optimisation process. 
automation of systems integrated by heterogeneous elements - resources is based on technologies that provide interoperability, i.e. "open" systems architectures, which is additional requirement for market of resources operation, and $\mathrm{A} / \mathrm{V} \mathrm{E}$ design and operation as well.

As the market is intensively based in high-level information technologies (distributed software applications, information systems and databases, telematics applications, and Wide Area Networks), we are referring to an electronic and virtual market. The electronic and virtual market provides information about the candidate resources to integrate a virtual enterprise, about products and about clients. On the same way, the electronic and virtual market provides:

- access procedures,

- remote negotiation and utilisation of services, and

- interacts with existing networks and markets of suppliers and users of information, services and products.

We are now prepared to introduce the concept of Market of Resources, as an electronic and virtual market, subscribed by a subset of the universe of independent resources. To this subscription corresponds the formal description of the resources using a Resources Representation Language and its integration in a knowledge base. The organisational aspect of the Market of Resources consists on an electronically delivered intermediation service, between the candidate resources integrating the knowledge base and clients, which are organisations looking for resources to integrate.

Thus, we will say that the resources selection domain, to satisfy the tasks of the enterprise, within the same enterprise, i.e. company, represents the lower limit of the resources selection domain space. On the contrary, the global resources selection domain, to satisfy the tasks of the enterprise, implying the other enterprises subcontracting, represents the upper limit of the resources selection domain space. The global domain provides virtually, and from a practical point of view, an almost infinite resources selection domain size for the optimal organisation structure synthesis providing the highest level of competitiveness.

Figure 1, using an $\mathrm{IDEF}^{8}$ diagram, represents the overall process, from the Creation of a Market of Resources (Process A1.), to be used by the process of Design and Integration of the A/V Enterprise (Process A2.) that will answer to a market opportunity, i.e., to produce a product (Process A3.). This overall process exceeds the objectives of the present contribution, as we are solely concerned with the process of A/V E Design, working on a Market of Resources, and thus excluding the problematic of $\mathrm{A} / \mathrm{V}$ E Operation.

The diagram illustrates the structural relations between two processes and the entities present in the system. The processes (represented as boxes) transform the inputs into outputs (respectively the left and the right arrows of a process), using the mechanisms for the transformation (the bottom arrows of a process) and constrained by control information or conditions under which the transformation occurs (the top arrows).

8 ICAM DEFinition methodology (ICAM - Integrated Computer-Aided Manufacturing) 


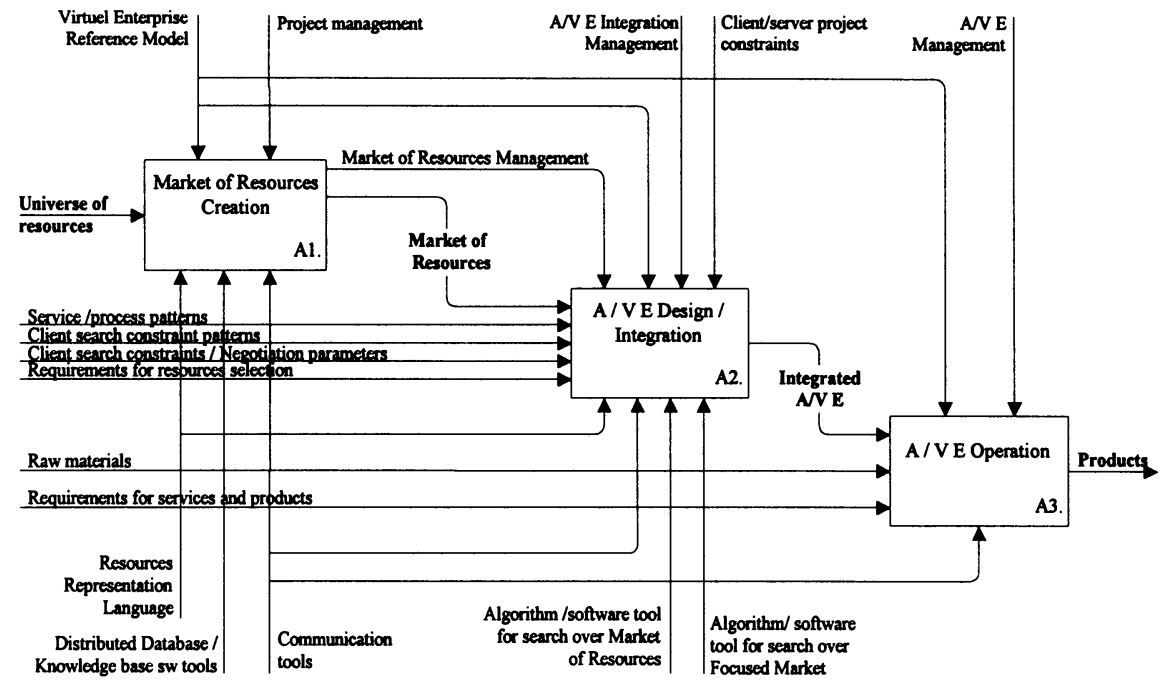

Figure 1 - IDEF0 representation of the global process for the creation of a Market of Resources and for A/V E design, integration, and operation

\section{THE NEED OF A FOCUSED MARKET OF RESOURCES}

To keep the dynamics of the Agile /Virtual Enterprise model, i.e. the flexibility of the enterprise organisation, the optimal search should be obtained almost in real time. The search effort, inherent to a search algorithm, to find the optimal selection of resources grows exponentially with the domain size (i.e. the search effort is of the exponential complexity in function of the domain size. In other words, the search problem is, for the general case, by nature, a NP class problem.), and thus is classified as an intractable problem.

The complexity of the resources selection in general means that a compromised domain size (as a base for the solution space construction) should be used for each resource search. This concept will be designated by Focused Market of Resources. We define the concept of Focused Market of Resources as a subset of the Market of Resources where a given search for an independent resource is to take place.

The proposal of a Focused Market approach, complemented by automatic search over the knowledge base, makes possible to solve the problem, if possible, almost in real time.

The Market of Resources is an universe (not the universe) of resources able to furnish a reasonable solution space.

In short, we have identified two market concepts:

- The Market of Resources, and

- The Focused Market of Resources. 


\section{ROLE AND CHARACTERISTICS OF THE FOCUSED MARKET OF RESOURCES}

The Focused Market of Resources is the output of the execution of an algorithm, having as input, besides the description of the pretended resource, some functional search parameters (time, money,... available for the search), introduced by the client of the service. Those parameters will determine the dimension of the Focused Market (subdomain space), as a subset of the Market of Resources. The search algorithm will work within the Focused Market and provide the best, or the best "with high probability", resource therein included.

As a consequence of the complexity associated with the optimal search, the constitution of the market for the integration of resources in Agile /Virtual Enterprises has for two requisites:

1) the identification of a focused domain;

2) the separation "in time and space" of:

i) the process of the focused domain identification, i.e. the focused domain recognition, and

ii) the search or selection process.

The first requisite derives from the fact that the search or selection effort (and hence search time) grows exponentially with the domain size (as well as it depends of the concrete search algorithm applied, Figure 2a). Another fact that implies the need for the focused domain is that the quality of the search result (match of the requirement and the resource identified) grows with the solution space, i.e. to the total quality corresponds an infinite search time. So, a compromised dimension is to be found, as Search time is a critical resource, as it is directly connected with either the search cost and with the dynamics of business opportunities. From the other side, the shape of the "quality function" is "convex", i.e. the grow rate of the function decreases (the second derivation of the function is negative) and probably it is not practical to extend the search time to much, Figure $2 \mathrm{~b}$.

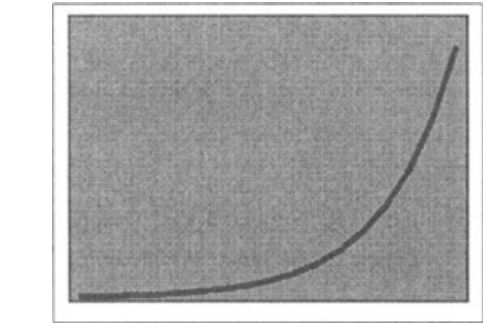

Search_effort $=f($ Domain size, Search_algorithm)

a)

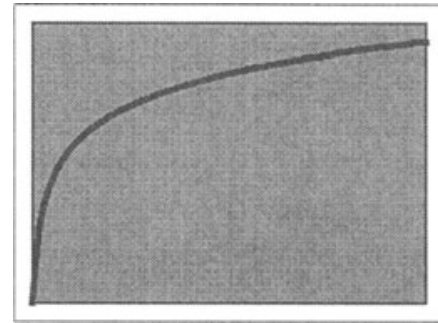

Quality $=f($ Search_effort $)$

b)

Figure 2-Complexity of a) the resources search effort in function of the domain size and the search algorithm and b) the quality in function of the search effort

A focused domain should be proposed for each search, reasonably dimensioned, to allow a good match, at a limited time.

The second requisite derives from the facts that there are two completely distinct processes; distinct either because of the kind of information processed and because 
of the dynamics associated with the entities involved in the integration of Agile / Virtual Enterprise.

The two processes outlined above, conducting to the optimal selection of resources to meet a client demand (the focused domain identification process and the search process) can be represented using the IDEF0 representation as in Figure 3.

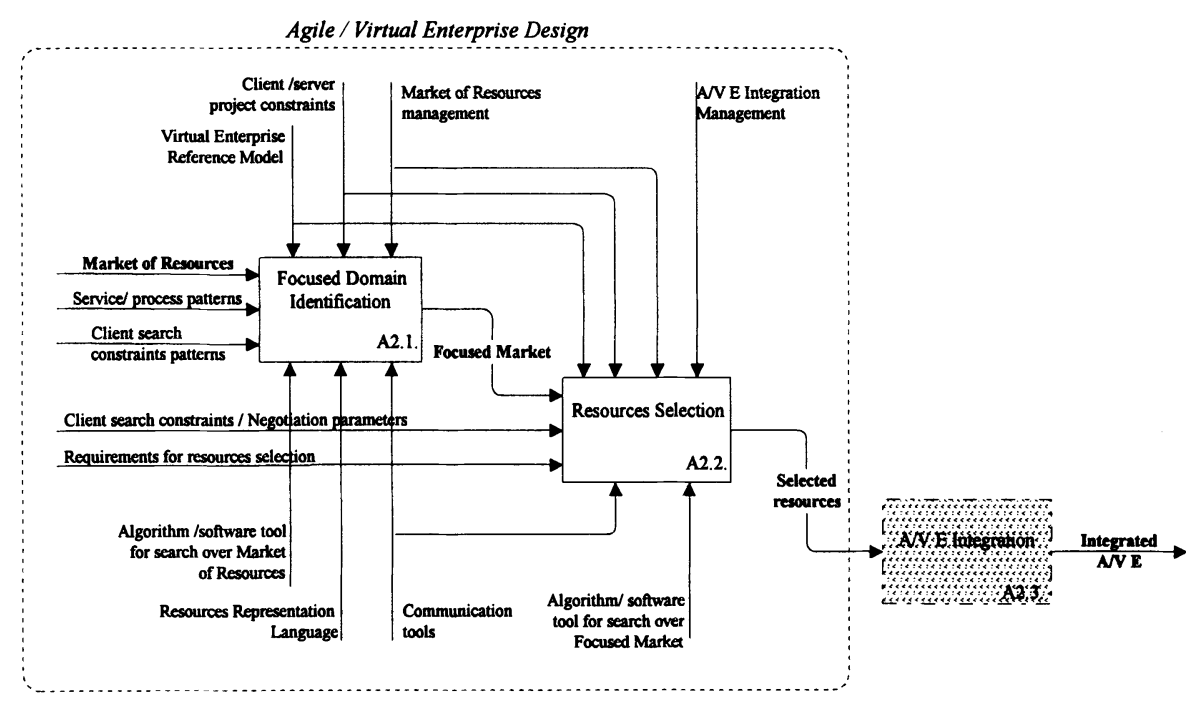

Figure 3 - IDEF0 representation of the resources selection processes for $A / V E$ integration

\section{The focused domain identification process (A2.1.)}

The main input is the information concerning the resources (primitive or complex resources) included in, or subscribed to, the Market of Resources (geographically distributed) database or knowledge base. The process consists on filtering the knowledge base (Market of Resources) into a Focused Market (composed by the candidate resources). The process should be performed off-line, i.e. before the requirement for the resources search for the concrete service, from the following reason. As we don't know in advance which one of resources present on the market is appropriate and which one is inappropriate, it is necessary to visit all (or as much as possible) resources on the market. If this process is performed after receiving the requirement for the resource search, due to the limited search time it could happen that we can't find any appropriate (or candidate) resource although it exists on the market (during the allowed search time we could visit by chance only inappropriate resources).

On the other side, as we have to identify the focused market before receiving the concrete requirement for the resource search, i.e. for a future, hypothetical requirement, the focused market identification process must be based on some requirement patterns, i.e. service or process patterns (of the concrete services or processes that will appear in the future for which the resource is required). The service/process patterns will determine the qualitative contents of the focused 
market. But the focused market contents does not depend on the service/process patterns only but also on the client's search constraints as, for example, the required quality, the negotiation constraints, the available time to identify the required resource and the cost the client is willing to pay for the service. These constraints will determine the focused market size. As the focused market identification process should be performed off-line, as we said above, it must be based on the client's search constraint patterns. In other words, as the process is supposed to be performed off-line, i.e. before the concrete requirement for the resource search, we should have some ideas about hypothetical types and constraints of client's requirements.

So, the second part of the input in the focused market identification process are service/process patterns and client search constraints patterns.

The practical consequence of this approach is that we will have identified a (knowledge base on the) set of focused markets that correspond to different service/process patterns and search constraints patterns, i.e. to different hypothetical types of requirements for resources. This set of focused markets is prepared in advance in order to provide efficient, on-line optimal (or optimised) concrete resource identification when the time comes.

The process is supported by an automatic search over the knowledge base (on the diagram, "Algorithm/software tool for search over domain Market of Resources") and by corresponding communication tools and protocols (on the diagram "Communication tools"). The process is constrained by two main kinds of control information: (1) the Market of Resources management rules and (2) the constraints of the focused market identification process itself (on the diagram "Client/server constraints").

The process output is the focused domain or the Focused Market, which is presented as an input to the next process (A2.2).

\section{The search process (A2.2.)}

The dimension and the quality of the focused domain that is proposed to this process are critical. The dynamics of the Agile / Virtual Enterprise model demands the selection to take place almost at real time.

The process corresponds to visiting all the elements proposed by the Focused Market, in order to identify negotiation parameters (availability, time to respond to the demand, or time to offer the resource, and costs), and perform an optimal search algorithm considering the client's negotiation parameters (defined by the candidate resources) and subjected to the client project constraints (time to complete the product, cost, etc.).

The search process consists on an automatic search algorithm (in the diagram, "Algorithm/software tool for search over Focused Market"), visiting all the candidate resources from the Focused Market supported by information and communication technologies (in the diagram, "Communication tools"), in order to identify the negotiation parameters and the availability of the resources, according to the requirements for resources selection (in the diagram, this information corresponds to the input flows "Negotiation parameters" and "Requirements for resources selection"). The search process is constrained by two kinds of control information: (1) the Market of Resources management rules and (2) the A/V E 
integration management. From this process will result a list of the best resources to satisfy the client requirements for resources to integrate the concrete $A / V$ E.

Implementation of both processes, $\mathrm{A} 2.1$ and $\mathrm{A} 2.2$, should follow the $\mathrm{A} / \mathrm{V} \mathrm{E}$ reference models.

\section{CONCLUSIONS}

The integration of resources in Virtual Enterprises as a dynamic process cannot cope efficiently with the complexity associated with a search of candidate resources from the universal mesh of unregulated and not normalised offer of resources available on the global market. We have proposed a new environment which should be considered in the research concerning the emergent paradigms of management and manufacturing, namely the Agile /Virtual Enterprise model. This new environment consists on the execution of automatic search under a subdomain of resources, called focused market of resources, with high potential to contain a solution, allowing to process requirements for resources in almost real time, preserving the desired business dynamics.

\section{REFERENCES}

1. Goldman S. L., Nagel R.. N., Preiss K. (1995). Agile competitors and virtual organizations, Van Nostrand Reinhold.

2. Maier, G., Traxler, H. (1995). "The Emergence of the Virtual Enterprise?", 35th European Congress of the Regional Science Association, Odense, Denmark, August 1995.

3. Mitsuishi M., Nagao T., Hatamura Y., Kramer B., Warisawa S. (1992). A Manufacturing system for the Global Age, in Olling G.J., Kimura F. (eds) Human Aspects in Computer Integrated Manufacturing, Elsevier Scientific.

4. NIIIP (1996). The NIIIP Reference Architecture, http:/www.niiip.org.

5. Onosata M., Iwata K. (1993). Development of a Virtual Manufacturing System by Integrating Product Models and Factory Models, Annals of the CIRP, Vol.42/1/1993, pp 475-478.

6. Prahalad, C. K. and Doz, Y. L. (1987). The Multinational Mission, New York Free Press.

7. Putnik G. D. (1997). Towards OPIM System, in M. A. Younis, S. Eid (Eds.) Proceedings of the 22nd ICC\&IE Conference - Computers \& Industrial Engineering, Cairo.

8. Putnik G. D., Sousa R. M., Moreira J. F., Carvalho J. D., Spasic Z., Babic B. (1998). Distributed/Virtual Manufacturing Cell: An Experimental Installation, in Proceedings of 4th International Seminar on Intelligent Manufacturing Systems, Belgrade. 